\title{
Double-auction-based energy trading for small cell networks with energy harvesting
}

Navid Reyhanian, Behrouz Maham, Vahid Shah-Mansouri, Chau Yuen

- School of Engineering

\begin{abstract}
In this paper, we propose a novel online centralized algorithm for enabling non-cooperative and energy harvesting capable base stations (BSs) to trade energy in multi-tier cellular networks. BSs are connected to the non-renewable energy source used by a BS when it cannot harvest enough energy to serve its connected users. A double auction trading framework is proposed to motivate BSs with the extra harvested energy to share their surplus energy with BSs that have not harvested sufficient energy. In addition, BSs with energy deficit are stimulated to buy surplus energy of other BSs which results in reducing of the non-renewable energy consumption. The algorithm satisfies truthfulness, individual rationalities and budget balance. Moreover, it reaches the Nash equilibrium. The extra harvested energy is distributed by the smart grid that prevents energy accumulation which results in the waste of the harvested energy due to limited battery capacities. To reduce smart grid usage in distributing energy, an optimization is embodied in the proposed algorithm to assign BSs with energy deficit to near BSs with extra harvested energy. Simulations results show that the non-renewable energy consumption reduces dramatically when the algorithm is applied. In addition, BSs gain more profit, consequently, their utility functions enhance.
\end{abstract}

\begin{tabular}{ll} 
Original language & \multicolumn{1}{l}{ English } \\
Title of host publication & 2016 IEEE International Conference on Communications, ICC 2016 \\
Publisher & $\frac{\text { Institute of Electrical and Electronics Engineers Inc. }}{9781479966646}$ \\
ISBN (Electronic) & Published - Jul 122016 \\
State & $\begin{array}{l}2016 \text { IEEE International Conference on Communications, ICC } 2016 \text { - } \\
\text { Kuala Lumpur, Malaysia }\end{array}$ \\
Event & 2016 IEEE International Conference on Communications, ICC 2016 \\
Other & Malaysia \\
Country & Kuala Lumpur \\
City & $5 / 22 / 16 \rightarrow 5 / 27 / 16$
\end{tabular}

Reyhanian, N., Maham, B., Shah-Mansouri, V., \& Yuen, C. (2016). Double-auction-based energy trading for small cell networks with energy harvesting. In 2016 IEEE International Conference on Communications, ICC 2016. [7511350] Institute of Electrical and Electronics Engineers Inc.. DOI: 10.1109/ICC.2016.7511350 